\title{
Regulation of Cytoplasmic Dynein ATPase by Lis1
}

\author{
Mariano T. Mesngon, ${ }^{1}$ Cataldo Tarricone, ${ }^{3}$ Sachin Hebbar, ${ }^{1}$ Aimee M. Guillotte, ${ }^{1}$ E. William Schmitt, ${ }^{2}$ Lorene Lanier, ${ }^{4}$ \\ Andrea Musacchio, ${ }^{3}$ Stephen J. King, ${ }^{2}$ and Deanna S. Smith ${ }^{1}$ \\ ${ }^{1}$ Department of Biological Sciences, University of South Carolina, Columbia, South Carolina 29208, 2Division of Molecular Biology and Biochemistry, \\ University of Missouri-Kansas City, Kansas City, Missouri 64110, 32Department of Experimental Oncology, European Institute of Oncology, 20141 Milan, \\ Italy, and ${ }^{4}$ Department of Neuroscience, University of Minnesota, Minneapolis, Minnesota 55455
}

Mutations in Lis1 cause classical lissencephaly, a developmental brain abnormality characterized by defects in neuronal positioning. Over the last decade, a clear link has been forged between Lis1 and the microtubule motor cytoplasmic dynein. Substantial evidence indicates that Lis 1 functions in a highly conserved pathway with dynein to regulate neuronal migration and other motile events. Yeast two-hybrid studies predict that Lis1 binds directly to dynein heavy chains (Sasaki et al., 2000; Tai et al., 2002), but the mechanistic significance of this interaction is not well understood. We now report that recombinant Lis 1 binds to native brain dynein and significantly increases the microtubule-stimulated enzymatic activity of dynein in vitro. Lis 1 does this without increasing the proportion of dynein that binds to microtubules, indicating that Lis 1 influences enzymatic activity rather than microtubule association. Dynein stimulation in vitro is not a generic feature of microtubule-associated proteins, because tau did not stimulate dynein. To our knowledge, this is the first indication that Lis 1 or any other factor directly modulates the enzymatic activity of cytoplasmic dynein. Lis 1 must be able to homodimerize to stimulate dynein, because a C-terminal fragment (containing the dynein interaction site but missing the self-association domain) was unable to stimulate dynein. Binding and colocalization studies indicate that Lis1 does not interact with all dynein complexes found in the brain. We propose a model in which Lis 1 stimulates the activity of a subset of motors, which could be particularly important during neuronal migration and long-distance axonal transport.

Key words: axon transport; dynein; lissencephaly; neuronal migration; microtubules; Lis1

\section{Introduction}

Lissencephalies are developmental abnormalities characterized by reduced brain folding, cognitive impairment, and progressively worsening epileptic seizures (Kato and Dobyns, 2003). Heterozygous disruption of the Lis 1 gene causes type 1 lissencephaly. Postmortem cytohistological studies indicate that neurons are aberrantly positioned in affected regions. Lis1 haploinsufficiency in mice results in defective interkinetic nuclear migration in the ventricular zone and slowed neuronal migration in the developing cortex (Hirotsune et al., 1998; Gambello et al., 2003). Total loss of Lis 1 is lethal in mouse embryogenesis (Hirotsune et al., 1998; Cahana et al., 2003).

Significant progress has been made in elucidating the cellular processes regulated by Lis1 (Vallee et al., 2001; Wynshaw-Boris and Gambello, 2001; Gupta et al., 2002; Xiang, 2003). Although Lis1 is enriched in the brain (Smith et al., 2000), Lis1 function is not restricted to the nervous system. Mutations in the highly conserved Lis1 protein profoundly impact migration, nuclear distribution, and mitosis in a range of organisms from yeast to mammals. However, the sensitivity of neurons to Lis1 reduction points to a unique importance in these cells, effecting not only

Received July 1, 2005; revised Jan. 13, 2006; accepted Jan. 14, 2006

This work was supported by National Institutes of Health Grant 1P20RR017698-01 (D.S.S. laboratory). S.J.K. was supported by an award from the American Heart Association.

Correspondence should be addressed to Deanna S. Smith, Department of Biological Sciences, University of South Carolina, Coker Life Sciences Building 607, 700 Sumter Street, Columbia, SC 29208. E-mail: deannasm@biol.s.c.edu. DOI:10.1523/JNEUROSCI.5095-05.2006

Copyright $\odot 2006$ Society for Neuroscience $\quad$ 0270-6474/06/262132-08\$15.00/0 migration and mitosis but neuritic structure, axon transport, and synaptic function (Liu et al., 2000; Williams et al., 2004).

The $\mathrm{C}$ terminus of Lis 1 folds to form a $\beta$-propeller as do many proteins with short 40 aa repeated motifs that end in Trp-Asp (WD) (Kim et al., 2004; Tarricone et al., 2004). Lis1 dimerizes through a self-association domain in the $\mathrm{N}$ terminus. Lis1 may interact directly with tubulin and/or microtubules (MTs) (Sapir et al., 1999). Although the list of potential Lis1-interacting proteins continues to grow, an important key to understanding the role of Lis 1 in development and disease is its association with a multisubunit motor protein, cytoplasmic dynein (Smith et al., 2000; Vallee et al., 2001; Wynshaw-Boris and Gambello, 2001; Gupta et al., 2002; Xiang, 2003), which has critical functions in intracellular transport, mitosis, migration, and nuclear positioning (Banks and Heald, 2001; Dujardin and Vallee, 2002; Tsai and Gleeson, 2005). Dynein performs microtubule-dependent ATP hydrolysis to translocate toward microtubule minus ends. In neurons, cytoplasmic dynein is the predominant motor for longdistance retrograde transport in axons (Goldstein and Yang, 2000) and has been linked to neurodegenerative diseases involving defects in transport (Hirokawa and Takemura, 2004). Dynein is a protein complex. Dynein heavy chains (DHCs) are ATPases, whereas dynein intermediate chains (DICs), dynein light chains (DLCs), and dynein light intermediate chains (LICs) confer cargo specificity and subcellular targeting. Despite intense scrutiny over the last decade, relatively little is understood about the mechanistic significance of the Lis1/dynein association. Our work, analyzing the impact of altering Lis1 expression in nonneuronal cells, strongly suggested a stimulatory role for Lis1 with 
A

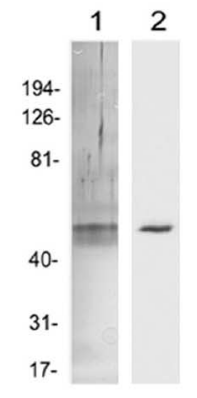

B

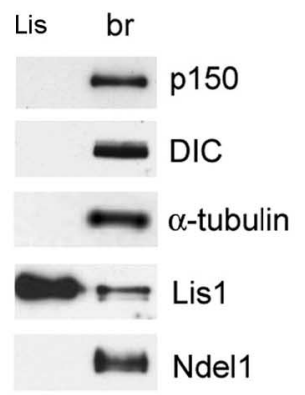

E

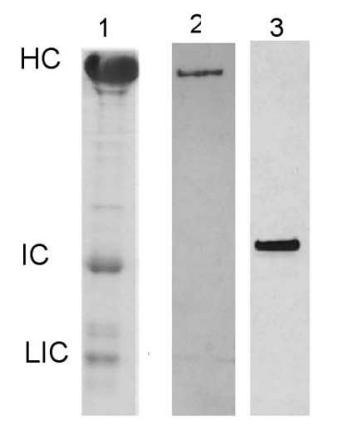

C

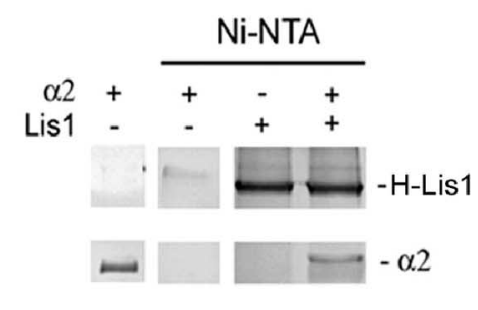

\section{Materials and Methods}

Antibodies. A rabbit IgG against Lis1 was used in Figure 5B (H300; Santa Cruz Biotechnology, Santa Cruz, CA); otherwise, an affinity-purified rabbit Lis1 antibody was used (Smith et al., 2000). Other antibodies used were as follows: mouse DIC and rabbit DHC antibodies (IgG 74.1, R-325; Santa Cruz Biotechnology) and mouse $\alpha$-tubulin (clone B-5-1-2; Sigma, St. Louis, MO).

Protein isolation. Full-length recombinant Lis1 and a fragment of Lis1 encompassing the WD domain (amino acids 51-410) were produced as histidine-tagged proteins in Sf9 insect cells and purified on Ni-NTA resin (Invitrogen, Carlsbad, CA) (see Fig. 1). The tag was cleaved using TEV (tobacco etch virus) protease, designed for use with the Ni-NTA system. The released Lis1 was dialyzed against Tris-buffered saline and then stored at $-20^{\circ} \mathrm{C}$ in $50 \%$ glycerol. Cytoplasmic dynein was purified from fresh bovine brains as described previously (Bingham et al., 1998). Single-use aliquots of dynein were stored frozen at $-80^{\circ} \mathrm{C}$. The $4 \mathrm{~L}$ longest splice variant of tau was expressed in bacteria and purified as described previously (Paudel and Li, 1999). Tau was flash frozen and stored at $-80^{\circ} \mathrm{C}$ until use.

ATPase assay. A thin-layer chromatography (TLC) assay (Gilbert and Mackey, 2000) was modified for use with our purified cytoplasmic dynein (see Fig. $2 A, B, D$ ). The amount of radiolabeled ADP generated after $30 \mathrm{~min}$ in the presence or absence of microtubules and/or Lis1 was used as an indication of enzymatic activity. Reaction volumes were $50 \mu \mathrm{l}$ in BrB80 ( $80 \mathrm{~mm}$ PIPES, 1 mм EGTA, 1 mm MgCl2). Equimolar amounts of purified Lis1 and dynein (5 pmol) were incubated at $37^{\circ} \mathrm{C}$ for $30 \mathrm{~min}$ with $10 \mu \mathrm{Ci}$ $\alpha-{ }^{32} \mathrm{P}$-ATP with or without $8 \mu \mathrm{M}$ paclitaxelstabilized microtubules. Microtubules were prepared from bovine brain tubulin (Cytoskeleton, Denver, CO) as described below. The reaction mixture was centrifuged at $13 \mathrm{~K} \mathrm{rpm}$ for $2 \mathrm{~min}$. Totals of $0.2-0.6 \mu \mathrm{l}$ of the supernatants were spotted onto PEI-Cellulose F TLC plates (EMD Chemicals, Gibbstown, NJ). TLC plates were developed in $0.6 \mathrm{~m}$ potassium phosphate buffer, $\mathrm{pH}$ 3.4. ATP and ADP spots were quantified densitometrically using Quantity One software (Bio-Rad, Hercules, CA) and by scintillation counting of spots excised from TLC plates.

Comparison of microtubules in the presence of dynein alone or in the presence of both $d y$ nein and Lisl. Purified bovine tubulin (Cytoskeleton) was incubated at a concentration of $5 \mathrm{mg} / \mathrm{ml}$ in ice-cold BRB80 (80 mм PIPES, $1 \mathrm{mM} \mathrm{MgCl}_{2}, 1 \mathrm{~mm}$ EGTA, pH 6.8), $1 \mathrm{~mm}$ DTT, $1 \mathrm{~mm} \mathrm{GTP}$, and $2.5 \mu \mathrm{g}$ of rhodaminelabeled tubulin (Cytoskeleton) for $2 \mathrm{~min}$ at $0^{\circ} \mathrm{C}$ and then clarified in a Beckman TLA 120.2 rotor (Beckman Instruments, Fullerrespect to cytoplasmic dynein (Smith et al., 2000). We now report that Lis1 stimulates the average in vitro enzymatic activity of dynein by $\sim 40 \%$. Other evidence indicates that a subpopulation of brain dynein binds Lis1. Together, these data suggest that ATPase activity is substantially higher for dynein motors that interact with Lis1. We propose that modulation of the Lis1-dynein interaction serves as a control point in motor regulation. ton, CA) at $94,000 \mathrm{rpm}$ for $5 \mathrm{~min}$ at $4^{\circ} \mathrm{C}$. The clarified mix was incubated at $37^{\circ} \mathrm{C}$ for $2 \mathrm{~min}$. Taxol was added stepwise to equimolar concentrations $(5,50$, and $500 \mu \mathrm{M})$ at $1 / 10$ the volume of the clarified mix and incubated for $5 \mathrm{~min}$ at $37^{\circ} \mathrm{C}$ between each Taxol addition. Polymerized microtubules were pelleted through a warm $40 \%$ glycerol cushion in a Beckman TLA 120.2 rotor at $94,000 \mathrm{rpm}$ for $5 \mathrm{~min}$ at $37^{\circ} \mathrm{C}$. The microtubules were then washed in BRB80 and $50 \mu \mathrm{M}$ Taxol 
and resuspended in BRB80 and $50 \mu \mathrm{M}$ Taxol to a final tubulin concentration of $5 \mathrm{mg} / \mathrm{ml}$. Microtubules and dynein $+/-$ Lis 1 were incubated as described above for the ATPase assay, except that no radiolabeled ATP was included. Five microliters of the reaction mix were spotted onto a glass coverslip coated with polylysine to promote sticking. Coverslips were mounted using ProLong Gold (Invitrogen) and visualized with a plan-apo $63 \times / 1.40$ objective on an AxioVert 200 inverted microscope using a narrow bandpass filter (41004; Chroma Technology, Rockingham, VT).

Western blotting. Proteins separated by SDSPAGE and transferred to PVDF membranes were blocked in PBST (100 mm phosphate buffer, $\mathrm{pH}$ 8, $150 \mathrm{~mm} \mathrm{NaCl}, 0.1 \%$ Tween 20) containing $5 \%$ powdered milk and then incubated with indicated primary antibodies for $1 \mathrm{~h}$ at room temperature (see Figs. 1-4). Blots were probed with HRP-conjugated secondary antibodies (Pierce, Rockford, IL). HRP activity was detected using Western Lightning chemiluminescence reagents (Pierce).

Density sedimentation. Bovine brain dynein ( 5 pmol) was incubated with 15 pmol of Lis 1 for $30 \mathrm{~min}$ at $37^{\circ} \mathrm{C}$ (see Fig. $3 A$ ). For controls, each protein was incubated alone under the same conditions. Standards were BSA (3.3S), catalase (11.4S), and thyroglobulin (19.1S). Proteins were layered onto $5-25 \%$ sucrose gradients and centrifuged at $130,000 \times g$ for $8 \mathrm{~h}$ at $4^{\circ} \mathrm{C}$. Sixteen $50 \mu \mathrm{l}$ fractions were collected. Western blots of fractions 3-12 were probed for Lis1, DIC, and DHC.

Immunoprecipitation of purified proteins. 1.8 pmol of dynein was incubated with 10 pmol of Lis1 for $30 \mathrm{~min}$ at $37^{\circ} \mathrm{C}$ in PHM-T buffer $(60$ mм PIPES, 25 mм HEPES, 4 mм $\mathrm{MgSO}_{4}$ 0.1\% Tween 20, pH 6.9) (see Fig. 3B-E). For controls, each protein was incubated alone. Samples were exposed to DIC or Lis 1 antibodies for $1 \mathrm{~h}$ at $4^{\circ} \mathrm{C}$. Protein A-Sepharose (Zymed, San Francisco, CA) was added, and after rocking for $1 \mathrm{~h}$ at $4^{\circ} \mathrm{C}$, beads were washed three times in $150 \mathrm{~mm}$ phosphate buffer containing $200 \mathrm{~mm} \mathrm{NaCl}, 0.1 \%$ Tween 20 , and 0.1 or $1.0 \%$ Triton X-100.

Immunoprecipitation from rat tissues. Extracts from brain, liver, and testes of 3-week-old male rats were dounce-homogenized in lysis buffer (50 mм HEPES, pH 7.0, $150 \mathrm{~mm} \mathrm{NaCl}, 0.1 \% \mathrm{NP}-40,2 \%$ protease inhibitor cocktail III; Calbiochem, La Jolla, CA) at $4^{\circ} \mathrm{C}$ (see Fig. 4). Lysates were clarified at $28,000 \times g$ for $25 \mathrm{~min}$ and then incubated for $1 \mathrm{~h}$ with 74.1 at $4^{\circ} \mathrm{C}$, followed by $1 \mathrm{~h}$ with protein $\mathrm{A}$-Sepharose at $4^{\circ} \mathrm{C}$. Beads were washed three times in lysis buffer with $0.1 \%$ Tween 20 and $0.1 \%$ Triton $\mathrm{X}-100$ and then resuspended in $1 \times$ sample buffer.

Copelleting of dynein with microtubules in the presence or absence of Lis1. Taxol-stabilized microtubules were prepared in the same manner as described for the ATPase assay (see Fig. 2C). 2.5 pmol of Lis 1 or Dynein (or both) was incubated with $2 \mu \mathrm{M}$ Taxol, 2 mM GTP, 1 mM ATP, $8 \mu$ M casein, and $8 \mu \mathrm{M}$ microtubules at $33^{\circ} \mathrm{C}$ for $20 \mathrm{~min}$. Samples were pelleted through a $40 \%$ glycerol cushion at $37^{\circ} \mathrm{C}$ for $5 \mathrm{~min}$ at $94,000 \mathrm{rpm}$ in a Beckman TLA120.2 rotor. The pellets were resuspended in BRB80 and then placed on ice for $20 \mathrm{~min}$ to disrupt microtubules. The proteins in the soluble and pelleted fractions were concentrated by chloroformmethanol precipitation (Wessel and Flugge, 1984). The samples were analyzed by SDS-PAGE and Western blotting.

Analysis of Lis1 to dynein ratio in the insoluble fraction of mouse tissues. Mouse tissues were homogenized in lysis buffer as described above (see Fig. $4 C$ ). After centrifugation at $28,000 \times g$, the pellets were resuspended in $1 \times$ SDS sample buffer. A proportional amount of soluble and insoluble fractions were analyzed. For example, the brain sample was lysed in $0.5 \mathrm{ml}$, and the insoluble pellet was resuspended in $0.1 \mathrm{ml}$. Five percent of the soluble fraction and $20 \%$ of the insoluble fraction were loaded on the same gel. The ratio of insoluble to soluble protein obtained by densitometry was then multiplied by four to obtain an estimate of the true distribution.

Cell culture, fixation, and immunofluorescence. Cos-7 cells maintained in DMEM with $10 \%$ fetal calf serum, L-glutamine, and penicillin and streptomycin were plated onto $12 \mathrm{~mm}$ glass coverslips in 24-well plates (see Fig. 5). Cells were exposed for $1 \mathrm{~min}$ to $0.25 \%$ Triton X-100 and 1.0 $\mu \mathrm{M}$ paclitaxel in PHEM buffer (120 mM PIPES, 50 mM HEPES, $20 \mathrm{~mm}$ EGTA, 4 mm magnesium acetate, pH 6.9) and then fixed in 3\% paraformaldehyde for $20 \mathrm{~min}$, followed by $4 \mathrm{~min}$ in cold $100 \%$ methanol. The cell in Figure $5 B$ was fixed in cold $100 \%$ methanol for $2 \mathrm{~min}$. Hippocampi were dissected from embryonic day 17 (E17) rats essentially as described by Goslin et al. (1998). Dissociated neurons were plated onto glass coverslips coated with $1 \mathrm{mg} / \mathrm{ml}$ poly-D-lysine and incubated for $48 \mathrm{~h}$. Neurons were fixed in $2 \%$ paraformaldehyde for $15 \mathrm{~min}$ and then permeabilized for 10 min in PHEM buffer containing 0.1\% Triton X-100. Neurites were considered axons when twice as long as other processes and with growth cones twice the diameter of the soma (Deitch and Banker, 1993). After $1 \mathrm{~h}$ in block (PHEM with 10\% normal goat serum and 3\% bovine serum albumin), coverslips were incubated with primary antibodies and 
A

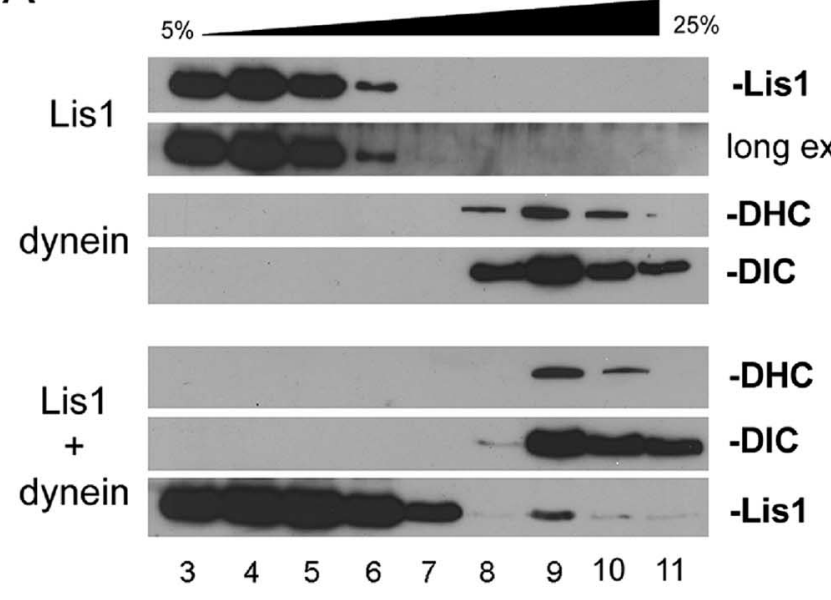

D

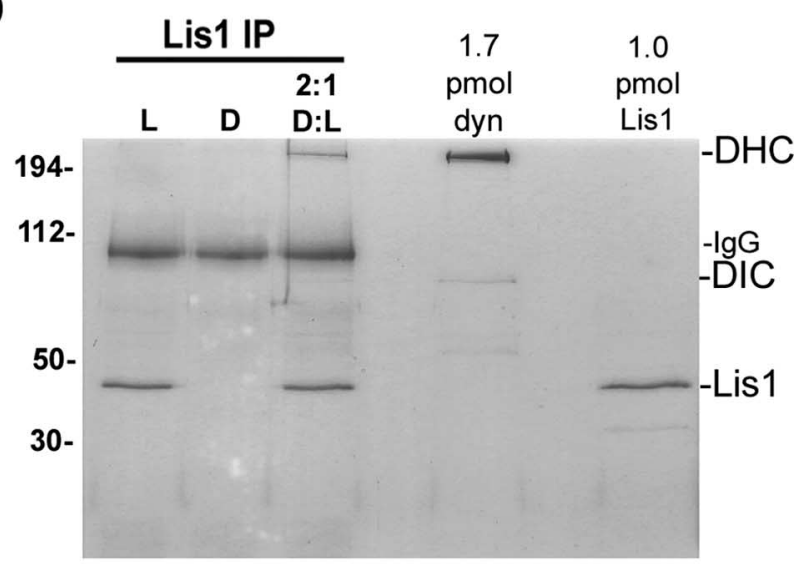

B

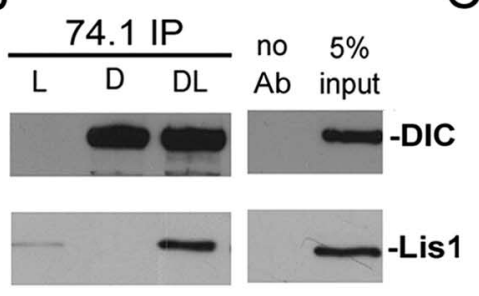

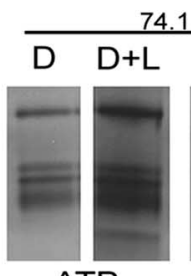

ATP
$74.1 \mathrm{IP}$

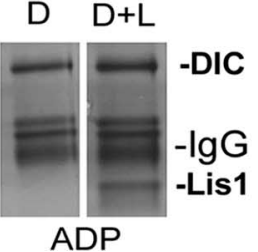

$3.0 \quad 1.0$ pmol pmol

Lis1 dyn
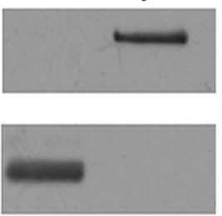

Lis1 IP

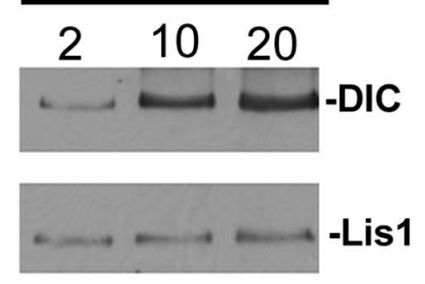

Figure 3. Lis 1 binds to a subset of cytoplasmic dynein motors in vitro. $A$, Fifteen picomoles of Lis1, 5 pmol of dynein, or a mixture of the two were loaded onto separate $5-25 \%$ sucrose gradients, as indicated (left). Fractions were probed with Lis1, DIC, or DHC antibodies as indicated (right). In the combined sample, $\sim 1.7 \mathrm{pmol}$ of Lis1 had shifted to higher fractions, where it cofractionated with DIC and DHC. No Lis1 was found in denser fractions in the absence of dynein, even when the blot was exposed overnight (long exp.). B, One picomole of dynein coimmunoprecipitated $\sim 0.37$ pmol of Lis1. L, Lis1; D, dynein; DL, Lis1 and dynein; No Ab, no antibody control; 5\% input, 5\% of DL. C, No difference was observed when 74.1 IPs were performed in the presence of excess ATP or ADP. D, The amount of DHC and DIC coprecipitating with Lis1 (L) when dynein (D) is present at a twofold molar excess is visualized by Coomassie staining. IgG is an antibody band. $E$, Left, Purified Lis1 and dynein (dyn) probed with Lis1 and dynein antibodies. Right, 2.5 pmol of Lis1 was incubated with 2, 10, and 20 pmol of purified dynein and then precipitated with Lis1 antibodies (Lis1 IP). Western blots were probed for Lis 1 and DIC. The ratio of Lis 1 to dynein in each IP was $0.37,1.2$, and 1.3, indicating that dynein binding had saturated when 10 pmol was present. All experiments were repeated with similar results. Representative blots or gels are shown.

then FITC- or Texas Red-conjugated goat $\alpha$-rabbit or $\alpha$-mouse IgG. Nuclei were labeled with $50 \mu \mathrm{g} / \mathrm{ml}$ bizbenzamide for $5 \mathrm{~min}$. Coverslips were mounted using Prolong Gold (Invitrogen).

Fluorescence microscopy. Fluorescent cells were visualized with a Zeiss (Thornwood, NY) Axiovert 200 inverted microscope using a plan-neo objective $(100 \times / 1.30)$ or a plan-apo $63 \times / 1.40$ objective (see Fig. 5). HQ series Chroma Technology filters sets (41004 and 41001) were used for double labeling with FITC- or Texas Red-conjugated secondary antibodies because of the steeper passbands and decreased bleedthrough. A standard filter set (31000) was used for bizbenzamide staining. Digital images were acquired with an AxioCam HR CCD camera and AxioVision 4.3 software.

Estimating the degree of Lis 1 and dynein colocalization in Cos7 cells. Using the $63 \times$ objective, $10-16$ optical sections were obtained from interphase Cos7 cells stained for Lis1 and DIC with the AxioVision 4.3 data acquisition module and the AxioVert 200 motorized microscope (see Fig. $5 B$ ). Images were deconvolved using a combined iterative algorithm supplied with the AxioVision 3D deconvolution module. A volume rendering was generated using the AxioVision Inside4D module, and an image was saved as a TIFF file for subsequent analysis in Adobe Photoshop (Adobe Systems, San Jose, CA). The punctate nature of overlapping Lis1 and dynein signals in one of these TIFF images is shown in Figure $5 B$. To simplify counting of overlapping and nonoverlapping puncta, yellow (overlapping) pixels were selected in Adobe Photoshop and replaced with a blue color. The image was then inverted, so the background became white, and overlapping and nonoverlapping puncta were more readily visualized (see Fig. 5B). Four images, containing three cells each, were printed. These were analyzed by manually counting using gridlines as shown in Figure 5B. The total number of green (dynein), orange (Lis1), and blue (both) spots was determined for each grid area. These were used to calculate the average percentage of dynein-positive puncta that were also positive for Lis1. For each data point, 12 cells were analyzed.

\section{Results}

\section{Lis1 stimulates dynein enzymatic activity in vitro}

To determine whether Lis1 could modulate dynein enzymatic activity, we used an in vitro thin-layer chromatography assay with purified dynein and recombinant Lis1. Figure 1 shows the characterization of the isolated proteins used throughout this study. Cytoplasmic dynein exhibited modest activity, $\sim 68( \pm 9) \mathrm{nmol}$ ATP hydrolyzed/mg of dynein/min (Fig. 2 A). As expected from published results, dynein activity was increased fourfold by the addition of stabilized microtubules to $281( \pm 19) \mathrm{nmol} / \mathrm{mg} / \mathrm{min}$. Lis1 alone did not possess any intrinsic enzymatic activity, but the addition of equimolar Lis1 increased the microtubule-stimulated ATPase activity of dynein by $\sim 40 \%$, to $389( \pm 15) \mathrm{nmol} / \mathrm{mg} / \mathrm{min}$. Doubling the Lis1 concentration did not further augment activity, whereas reducing the Lis1 concentration by $50 \%$ resulted in a smaller stimulation, indicating that the assay was performed within the linear range for Lis1 activity (Fig. 2B). The recombinant Lis1 used in our assay was properly folded, because the same Lis1 preparation was used for x-ray crystallography (Tarricone et al., 2004). Moreover, we found that Lis1 bound at the expected stoichiometry to PAFAH1b $\alpha 2$, a known binding partner (Fig. $1 C$ ).

Lis1 reportedly interacts with tubulin and possibly with microtu- 


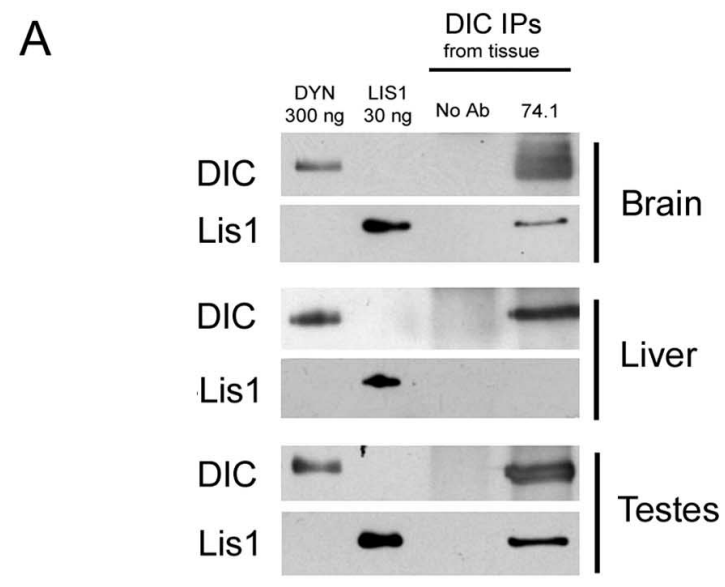

B

\begin{tabular}{|l|r|r|r|}
\hline Sample & \multicolumn{1}{|c|}{ brain } & \multicolumn{1}{c|}{ liver } & \multicolumn{1}{c|}{ testes } \\
\hline pmole dynein in 74.1 IP & 5.6 & 4.0 & 2.0 \\
\hline pmole Lis1 in 74.1 IP & 0.2 & ND & 0.2 \\
\hline Ratio Lis1/dynein in 74.1 IP & 0.04 & ND & 0.1 \\
\hline
\end{tabular}

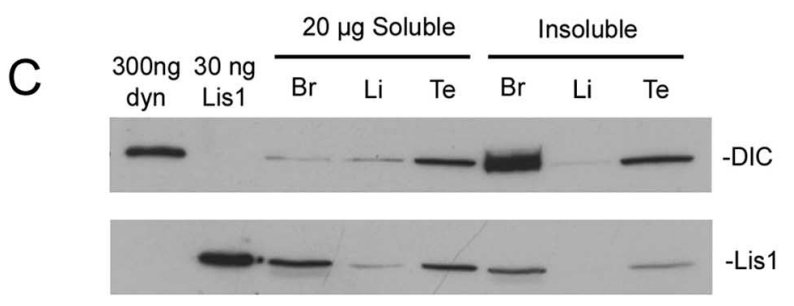

Figure 4. Lis1 associates with a subset of dynein complexes in tissue extracts. $A$, Dynein was immunoprecipitated from $10 \mathrm{mg}$ of adult rat extract prepared from the indicated tissues using the $74.1 \mathrm{DIC}$ antibody. Br, Brain; Li, liver; T, testes. Precipitated proteins were visualized by Western blotting using Lis1 and DIC antibodies. DYN, Purified dynein; Lis1, recombinant Lis1. B, The data in $\boldsymbol{A}$ were quantified by densitometry, comparing precipitated bands to known amounts of each protein. The molar amounts were calculated using the Lis1 dimer molecular weight of $90 \mathrm{kDa}$ and dynein molecular weight of $1.2 \mathrm{MDa}$. C, The amount of Lis1 and dynein in soluble extracts and insoluble pellets was compared for each tissue (see Materials and Methods). All experiments were repeated with similar results. Representative blots or gels are shown.

bules (Sapir et al., 1999). We reasoned that Lis1 could promote dynein association with microtubules, or alter microtubules themselves, to indirectly increase ATP hydrolysis. Two pieces of data indicate that this is not the case. First, Lisl did not increase the percentage of dynein that bound to microtubules (Fig. 2C) (see Materials and Methods). Second, we observed no Lis1-dependent change in MT length or bundling (Fig. $1 G$ ). Another concern is that any microtubule-associated protein (MAP) would stimulate dynein in vitro. Another microtubule binding protein, tau, has been shown to influence axon transport (Seitz et al., 2002). We therefore tested recombinant, full-length tau in our assay. Tau did not stimulate dynein activity, indicating that the Lis1 effect is specific. In fact, tau significantly reduced the impact of microtubules on dynein activation (Fig. 2D).

We next determined whether the C-terminal domain of Lis1, which contains a putative dynein binding domain but not a selfassociation domain (Tarricone et al., 2004), would be sufficient to stimulate dynein. If so, it would indicate that Lis1 dimerization is not critical for this function. A polypeptide encompassing the C-terminal domain of Lis1 was isolated and tested in the ATPase assay (Fig. 2D). Although this fragment binds dynein, it was not able to stimulate dynein enzymatic activity. Moreover, the fragment reduced stimulation by full-length Lis1, indicating that it competed for binding to dynein to block stimulation by Lis 1 dimers (Fig. 2D). Based on these findings, we propose that Lis 1 dimers are required for dynein stimulation.

\section{Lis1 binds a subset of dynein motors in vitro}

The significance of the increase in ATP hydrolysis depends on the percentage of functional motor complexes that actually bind Lis1. If all motors interact with Lis1, then Lis 1 probably activates each motor by $\sim 40 \%$. In contrast, if only a subset of motors bind Lis 1, 40\% could be an underestimate of the "per motor" stimulation. The actual increase would depend on the percentage of motors that bind Lis1. We used two approaches to estimate the interaction between Lis1 and dynein in vitro. First, the sedimentation of a Lis $1 /$ dynein mixture in a $5-25 \%$ sucrose gradient was compared with that of each protein individually and to sedimentation controls. In the absence of dynein, all of Lis1 was found in lower density fractions corresponding to a $\sim 7 \mathrm{~S}$ complex (Fig. $3 A$ ). Dynein alone was found in denser fractions corresponding to a $\sim 20 \mathrm{~S}$ complex. When $5 \mathrm{pmol}$ of dynein was mixed with a threefold molar excess of Lis 1 , some of the Lis 1 shifted to the $20 \mathrm{~S}$ portion of the sucrose gradient, where it cofractionated with cytoplasmic dynein. The signal density of the precipitated Lis 1 was compared with known amounts of recombinant Lis1. Only 1.67 pmol of Lis 1 cosedimented with 5 pmol of dynein, so Lis1 may bind approximately one-third of the motors.

Comparable results were obtained when dynein was immunoprecipitated from a mixture of Lis 1 and dynein (Fig. 3B). On average, $0.37 \mathrm{pmol}$ of Lis 1 precipitated with $1 \mathrm{pmol}$ of dynein. This was not increased by additional Lis 1 . To determine whether the degree of interaction was modulated by the nucleotidebinding status of the motor, we performed immunoprecipitations in the presence of excess ATP or ADP. We found no difference in the capacity of dynein to interact with Lis1 (Fig. 3C), indicating that Lis1 binds to dynein throughout the ATP mechanochemical cycle.

We also performed immunoprecipitations from the dynein/ Lis1 mix using a Lis1 antibody. When present in equimolar amounts, $0.37 \mathrm{pmol}$ of dynein coprecipitated per pmol of Lis 1 (Fig. 3D). Lis1 binding was saturated in the presence of fourfold excess dynein; the ratio of Lis1:dynein was 1.2 and 1.3 in the immunoprecipitations (IPs) with fourfold and eightfold excess dynein, respectively (Fig. $3 E$ ). The data indicate that each Lis 1 is competent to bind dynein, and that Lis1 dimers bind to a single dynein complex. They further support the hypothesis that approximately one-third of the dynein motors are competent to bind to Lis1 in vitro.

\section{Lis1 interacts with a subset of dynein motors in rat tissues}

To determine whether the ability of Lis1 to interact with a subset of dynein complexes is also true in vivo, we immunoprecipitated dynein from three different adult rat tissue lysates and then estimated the amount of coprecipitated Lis1 compared with a recombinant Lis 1 standard (Fig. $4 A, B$ ). Approximately 5.6 pmol of dynein coprecipitated $0.2 \mathrm{pmol}$ of Lis 1 from brain extracts. This 1:28 molar ratio is lower than that observed in vitro with purified proteins. Only $2.0 \mathrm{pmol}$ of dynein was precipitated from testes lysate, but this was associated with 0.2 pmol of Lis 1, a 1:10 molar ratio. No Lis1 was detected in the IP from liver, despite there being $4 \mathrm{pmol}$ of dynein in the precipitate. Thus, different tissues 
A
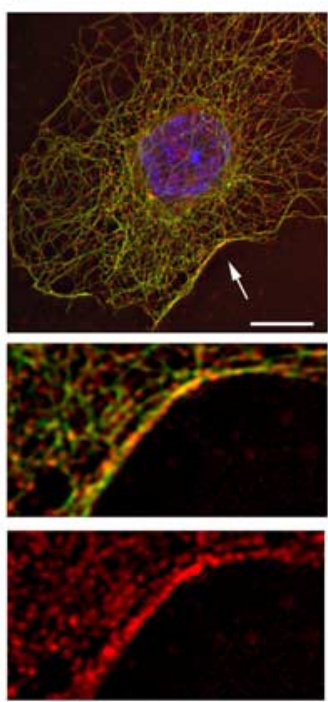

C
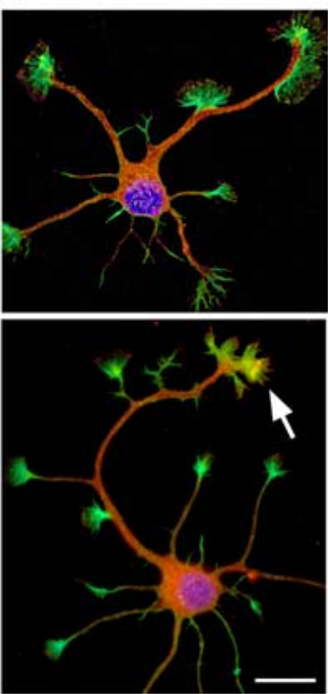

B
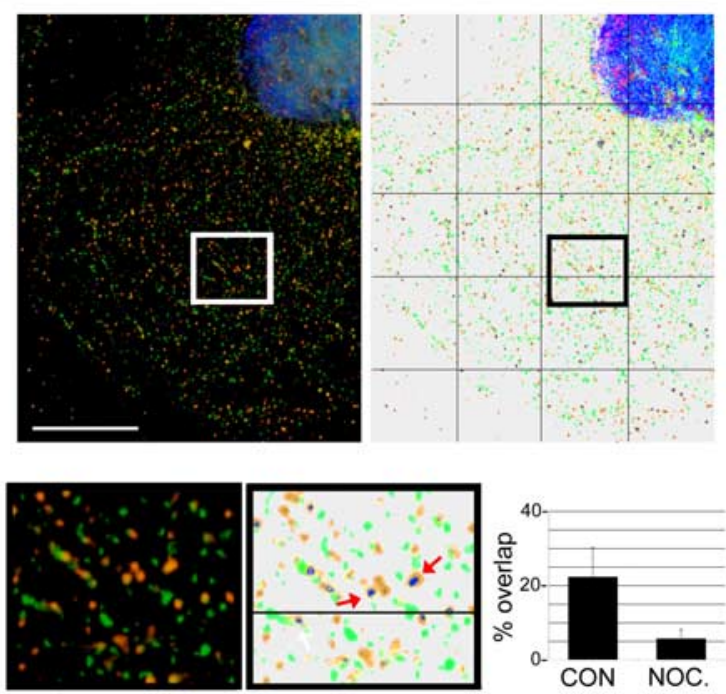

D
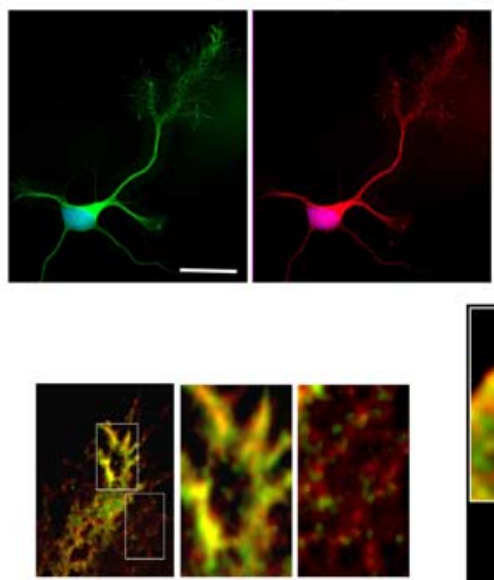

Figure 5. Partial colocalization of Lis1 and dynein. $\boldsymbol{A}$, Punctate Lis1 immunoreactivity (red) decorates microtubules (green). Bottom, An enlargement of the region indicated by the arrow. $\boldsymbol{B}$, Left, This pattern was also seen with Lis1 (red) and dynein (74.1-green), but the overlap was not extensive. The bottom left panel shows an enlargement of the area in the white square. Right, To more easily quantify overlap (yellow), deconvolved volumes were inverted and the hues adjusted so that overlapping puncta were colorized blue (arrows). The bottom middle panel shows an enlargement of the area in the black square. A bar graph shows the percentage of dynein puncta in control (CON) and nocodazole-treated (NOC.) cells that were also positive for Lis1 (12 cells \pm SD). C, Lis1 immunoreactivity (red) and actin staining (green) in E17 hippocampal neurons. Top, A stage 2 neuron with undifferentiated neurites. Bottom, A stage 3 neuron with a newly differentiated axonal growth cone enriched in Lis1 (arrow). D, Top, Dynein (red) and Lis1 (green) immunofluorescence in a stage 4 neuron. Bottom panels show enlargements of the growth cone (inset) and the axonal region indicated by the arrow. Two distinct regions in the growth cone are further enlarged (1 and 2 ). Scale bars: $\boldsymbol{A}, \boldsymbol{B}, \boldsymbol{D}, 10 \mu \mathrm{m} ; \boldsymbol{C}, 5 \mu \mathrm{m}$.

show a different degree of Lis1/dynein interaction, but in all cases, the proportion of dynein associated with Lis1 was less than that observed in vitro.

Because all of the active dynein may not be present in our tissue lysates, we compared the absolute molar ratio of Lis1 to dynein in insoluble pellets (Fig. 4C) (see Materials and Methods). Brain contained the highest proportion of insoluble dynein, followed by testes and then liver. Insoluble Lis 1 was present at an $\sim 1: 3$ molar ratio with dynein in each tissue. This suggests that in
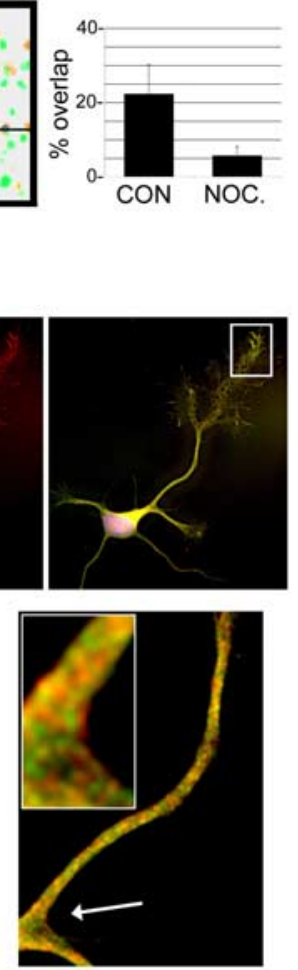

the insoluble fraction, no more than onethird of dynein motors are associated with Lis1.

Colocalization of Lis1 and dynein along microtubules and in growth cones is variable

Lis 1 immunoreactivity is enriched at centrosomes and in punctate structures that decorate microtubules in Cos-7 cells (Smith et al., 2000) (Fig. 5A). A similar pattern is observed using the 74.1 DIC antibody (Fig. 5B). To determine whether punctate MT-associated Lis1 is associated with dynein motors, we costained cells for Lis1 and DIC. In general, the punctate pattern of dynein staining was distinct from the punctate Lis1 pattern, although both were observed along the straight and curvilinear paths reminiscent of microtubules (Fig. 5B, left). When the images were digitally enhanced to increase the visibility of overlap puncta (see Materials and Methods), single and double-labeled puncta could be readily counted (Fig. $5 B$, right). On average, only $22 \%( \pm 7.9)$ of the dynein-positive puncta were also positive for Lis1. Although this analysis does not provide quantitative information at the molecular level, it supports the idea that only a subset of active dynein molecules interact with Lis1. Interestingly, treatment of Cos-7 cells with $10 \mu \mathrm{M}$ nocodazole to disrupt microtubules reduced the overlap to $5.6 \%$ $( \pm 2.5)$, indicating that some of the overlapping puncta were dependent on the presence of a microtubule cytoskeleton.

Lis 1 is present in neurites and cell bodies in developing stage 2 hippocampal neurons (Fig. 5C, top). Lis1 later accumulates in the newly emerging axonal growth cone (Fig. 5C, bottom, arrow) but not in minor processes. Dynein and Lis1 colocalize in cell bodies, neurites, and nascent axonal growth cones (Fig. 5D). A closer view reveals regional variation in the extent of overlap in neurites and in nascent growth cones, whereas axonal overlap seems fairly robust and consistent. This suggests that the interaction between Lis 1 and dynein may be dynamic and highly regulated in growth cones, and less so in axons.

\section{Discussion}

This work establishes Lis1 as a dynein regulatory protein and links its function to dynein enzymatic activity. The simplest interpretation of our data is that Lis1 acts specifically and directly on dynein. Although the increase in ATPase activity is modest at first glance, our determination that only one-third of the dynein molecules interacted with Lis 1 suggests that there were two populations of motors present in the ATPase assay. The major population did not stably bind Lis1 and is not expected to have had any change in ATPase activity, whereas a 
smaller population binds Lis1, resulting in a boost in ATPase activity. In this scenario, the enzymatic activity of the dynein as a whole would have increased by only $40 \%$, but the activity of Lis1-associated motors may have increased by $\sim 100 \%$.

The finding that Lis1 can stimulate dynein in vitro supports our model that Lis1 has an activating influence on dynein in cells (Smith et al., 2000). In our previous work, we found that raising Lis1 expression levels above that normally found in nonneuronal cells produced phenotypes suggestive of more active motors, whereas decreasing Lis1 expression had the opposite effect. Although altering Lis1 levels could have stimulated or inhibited neuron-like functions of dynein, our new data support a model in which changing the Lis1:dynein ratio in cells alters the proportion of dynein that is "stimulated." Brain and testes contain more Lis 1 and more dynein than the liver. The fact that brain and testes also express more Lis1 relative to dynein indicates that these tissues contain a higher proportion of Lis1-stimulated dynein.

The WD domain of Lis1 was not able to activate dynein in vitro and acted in a dominant-negative manner when presented along with full-length Lis1. This is particularly interesting in light of a report describing the phenotype of mice carrying a mutant Lis 1 allele and expressing a truncated Lis 1 protein similar to our WDLis1 (Cahana et al., 2001). Homozygotic expression of this protein in mice was lethal during the implantation stage, indicating that dimer formation is important for Lis1 function. However, heterozygotes, which expressed both the truncated protein and full-length Lis1, had developmental brain abnormalities that were somewhat less severe than mice with a null mutation in one allele. This could mean that in mice, the WD domain does not block activation of dynein by full-length Lis1, and that it may perform some Lis 1 functions. Alternatively, cells may have mechanisms to compensate for this truncated protein that are not available in vitro, such as sequestration.

One issue raised by our studies is the nature of events that regulate the interactions between Lis1 and dynein. Because dynein is heterogeneous, containing the range of isoforms present in the adult brain, we suspect that Lis 1 may preferentially bind to a specific class of dynein. This could involve posttranslational modification of dynein or Lis1. We observed no difference in Lis1 binding to dynein treated with $\lambda$ phosphatase, so dynein phosphorylation is probably not the determining factor (data not shown). Our murine Lis1, which was overexpressed in an insect cell line, is less likely to be posttranslationally modified to the same extent as native brain protein. However, some differential modification of Lis 1 could conceivably impact binding to dynein. Another possibility is that Lis1 binding to dynein is blocked by other proteins; this is unlikely to occur in our in vitro assay in which we include reasonably purified proteins. An alternate explanation is that specific isoforms of nonheavy chain dynein subunits modulate Lis1 binding. Future studies will be needed to determine whether this is the case. It will also be important to determine which signaling pathways impact the association of Lis1 with dynein.

Another issue raised by our studies is the question of whether Lis1 binding to dynein provides a continuous stimulus or acts as a trigger. If the latter is true, it does not appear to do so by increasing the association of dynein with microtubules. A high Lis1/dynein ratio in the brain and testes could indicate that the interaction between these proteins needs to be continuously renewed to maintain the level of stimulation. Finally, our data in- dicate that different tissues may use the Lis1 dynein interaction differently.

Although the demonstration that Lis 1 can directly regulate dynein is extremely interesting, we have only begun to address the cause and effect relationships between Lis1-induced increases in dynein ATPase activity and changes in cell behavior. Regardless of how the interaction is regulated, selective stimulation of some dynein isoforms by Lis1 in the nervous system is likely to be important for retrograde axonal transport and neuronal migration, processes that are impaired by reduced Lis1 expression.

\section{References}

Banks JD, Heald R (2001) Chromosome movement: dynein-out at the kinetochore. Curr Biol 11:R128-R131.

Bingham JB, King SJ, Schroer TA (1998) Purification of dynactin and dynein from brain tissue. Methods Enzymol 298:171-184.

Cahana A, Escamez T, Nowakowski RS, Hayes NL, Giacobini M, von Holst A, Shmueli O, Sapir T, McConnell SK, Wurst W, Martinez S, Reiner O (2001) Targeted mutagenesis of Lis 1 disrupts cortical development and LIS1 homodimerization. Proc Natl Acad Sci USA 98:6429-6434.

Cahana A, Jin XL, Reiner O, Wynshaw-Boris A, O’Neill C (2003) A study of the nature of embryonic lethality in LIS1-/- mice. Mol Reprod Dev $66: 134-142$.

Deitch JS, Banker GA (1993) An electron microscopic analysis of hippocampal neurons developing in culture: early stages in the emergence of polarity. J Neurosci 13:4301-4315.

Dujardin DL, Vallee RB (2002) Dynein at the cortex. Curr Opin Cell Biol 14:44-49.

Gambello MJ, Darling DL, Yingling J, Tanaka T, Gleeson JG, Wynshaw-Boris A (2003) Multiple dose-dependent effects of Lis1 on cerebral cortical development. J Neurosci 23:1719-1729.

Gilbert SP, Mackey AT (2000) Kinetics: a tool to study molecular motors. Methods 22:337-354.

Goldstein LS, Yang Z (2000) Microtubule-based transport systems in neurons: the roles of kinesins and dyneins. Annu Rev Neurosci 23:39-71.

Goslin K, Asmussen H, Banker G (1998) Rat hippocampal neurons in lowdensity culture. In: Culturing nerve cells, Ed 2 (Banker G, Goslin K, eds), pp 339-370. Cambridge, MA: MIT.

Gupta A, Tsai LH, Wynshaw-Boris A (2002) Life is a journey: a genetic look at neocortical development. Nat Rev Genet 3:342-355.

Hirokawa N, Takemura R (2004) Molecular motors in neuronal development, intracellular transport and diseases. Curr Opin Neurobiol 14:564-573.

Hirotsune S, Fleck MW, Gambello MJ, Bix GJ, Chen A, Clark GD, Ledbetter DH, McBain CJ, Wynshaw-Boris A (1998) Graded reduction of Pafah1b1 (Lis1) activity results in neuronal migration defects and early embryonic lethality. Nat Genet 19:333-339.

Kato M, Dobyns WB (2003) Lissencephaly and the molecular basis of neuronal migration. Hum Mol Genet 12:R89-R96.

Kim MH, Cooper DR, Oleksy A, Devedjiev Y, Derewenda U, Reiner O, Otlewski J, Derewenda ZS (2004) The structure of the N-terminal domain of the product of the lissencephaly gene Lis1 and its functional implications. Structure (Camb) 12:987-998.

Liu Z, Steward R, Luo L (2000) Drosophila Lis1 is required for neuroblast proliferation, dendritic elaboration and axonal transport. Nat Cell Biol 2:776-783.

Paudel HK, Li W (1999) Heparin-induced conformational change in microtubule-associated protein Tau as detected by chemical cross-linking and phosphopeptide mapping. J Biol Chem 274:8029-8038.

Sapir T, Cahana A, Seger R, Nekhai S, Reiner O (1999) LIS1 is a microtubule-associated phosphoprotein. Eur J Biochem 265:181-188.

Sasaki S, Shionoya A, Ishida M, Gambello MJ, Yingling J, Wynshaw-Boris A, Hirotsune S (2000) A LIS1/NUDEL/cytoplasmic dynein heavy chain complex in the developing and adult nervous system. Neuron 28:681-696.

Seitz A, Kojima H, Oiwa K, Mandelkow EM, Song YH, Mandelkow E (2002) Single-molecule investigation of the interference between kinesin, tau and MAP2c. EMBO J 21:4896-4905. 
Smith DS, Niethammer M, Ayala R, Zhou Y, Gambello MJ, WynshawBoris A, Tsai LH (2000) Regulation of cytoplasmic dynein behaviour and microtubule organization by mammalian Lisl. Nat Cell Biol 2:767-775.

Tai CY, Dujardin DL, Faulkner NE, Vallee RB (2002) Role of dynein, dynactin, and CLIP-170 interactions in LIS1 kinetochore function. J Cell Biol 156:959-968.

Tarricone C, Perrina F, Monzani S, Massimiliano L, Kim MH, Derewenda ZS, Knapp S, Tsai LH, Musacchio A (2004) Coupling PAF signaling to dynein regulation: structure of LIS1 in complex with PAF-acetylhydrolase. Neuron 44:809-821.

Tsai LH, Gleeson JG (2005) Nucleokinesis in neuronal migration. Neuron 46:383-388.
Vallee RB, Tai C, Faulkner NE (2001) LIS1: cellular function of a diseasecausing gene. Trends Cell Biol 11:155-160.

Wessel D, Flugge UI (1984) A method for the quantitative recovery of protein in dilute solution in the presence of detergents and lipids. Anal Biochem 138:141-143.

Williams SN, Locke CJ, Braden AL, Caldwell KA, Caldwell GA (2004) Epileptic-like convulsions associated with LIS-1 in the cytoskeletal control of neurotransmitter signaling in Caenorhabditis elegans. Hum Mol Genet 13:2043-2059.

Wynshaw-Boris A, Gambello MJ (2001) LIS1 and dynein motor function in neuronal migration and development. Genes Dev 15:639-651.

Xiang X (2003) LIS1 at the microtubule plus end and its role in dyneinmediated nuclear migration. J Cell Biol 160:289-290. 